\title{
BMJ Open Publication practices of sub-Saharan African Cochrane authors: a bibliometric study
}

\author{
Lawrence Mbuagbaw (1) , ${ }^{1,2,3,4}$ Anel Schoonees, ${ }^{5}$ Joy Oliver, ${ }^{6}$ Dachi Arikpo, ${ }^{7}$ \\ Solange Durão, ${ }^{6}$ Emmanuel Effa, ${ }^{7}$ Ameer Hohlfeld, ${ }^{6}$ Tamara Kredo (D) , ${ }^{6}$ \\ Charles Shey Wiysonge (D) , 5,6 Taryn Young ${ }^{5}$
}

To cite: Mbuagbaw $L$, Schoonees A, Oliver J, et al. Publication practices of sub-Saharan African Cochrane authors: a bibliometric study. BMJ Open 2021;11:e051839. doi:10.1136/ bmjopen-2021-051839

- Prepublication history for this paper is available online. To view these files, please visit the journal online (http://dx.doi org/10.1136/bmjopen-2021051839).

LM and AS contributed equally.

Received 30 March 2021 Accepted 17 September 2021

Check for updates

(c) Author(s) (or their employer(s)) 2021. Re-use permitted under CC BY-NC. No commercial re-use. See rights and permissions. Published by BMJ.

For numbered affiliations see end of article.

Correspondence to Dr Lawrence Mbuagbaw; mbuagblc@mcmaster.ca

\section{ABSTRACT}

Introduction Cochrane Africa (https://africa.cochrane. org/) aims to increase Cochrane reviews addressing high priority questions in sub-Saharan Africa (SSA). Researchers residing in SSA, despite often drawing on Cochrane methods, training or resources, conduct and publish systematic reviews outside of Cochrane. Our objective was to investigate the extent to which Cochrane authors from SSA publish Cochrane and non-Cochrane reviews.

Methods We conducted a bibliometric study of systematic reviews and overviews of systematic reviews from SSA, first by identifying SSA Cochrane authors, then retrieving their first and last author systematic reviews and overviews from PubMed (2008 to April 2019) and using descriptive analyses to investigate the country of origin, types of reviews and trends in publishing Cochrane and non-Cochrane systematic reviews over time. To be eligible, a review had to have predetermined objectives, eligibility criteria, at least two databases searched, data extraction, quality assessment and a first or last author with a SSA affiliation.

Results We identified 657 Cochrane authors and 757 eligible systematic reviews. Most authors were from South Africa $(n=332 ; 51 \%)$, followed by Nigeria $(n=126 ; 19 \%)$. Threequarters of the reviews $(71 \%)$ were systematic reviews of interventions. The intervention reviews were more likely to be Cochrane reviews $(60.3 \%$ vs $39.7 \%$ ). Conversely, the overviews ( $23.8 \%$ vs $76.2 \%)$, qualitative reviews $(14.8 \%$ vs $85.2 \%$ ), diagnostic test accuracy reviews ( $16.1 \%$ vs $83.9 \%$ ) and the 'other' reviews ( $11.1 \%$ vs $88.9 \%$ ) were more likely to be non-Cochrane reviews. During the study period, the number of non-Cochrane reviews increased more than the number of Cochrane reviews. About a quarter of the reviews covered infectious disease topics.

Conclusion Cochrane authors from SSA are increasingly publishing a diverse variety of systematic reviews and overviews of systematic reviews, often opting for nonCochrane journals.

\section{BACKGROUND}

Systematic reviews are recognised as important sources of information for decision making. ${ }^{1}$ However, disparities in research capacity worldwide have raised concerns about regional capacity in conducting systematic reviews. ${ }^{1}$ Appraisal of global systematic reviews shows most systematic review networks are dominated

\section{Strengths and limitations of this study}

- This is the first study comparing Cochrane and nonCochrane publications from authors in sub-Saharan Africa.

- We collected data from a comprehensive database (Archie).

- Bibliometric analyses alone cannot fully explain the behaviours or preferences of authors.

by high-income countries (HICs) even though low-income and middle-income countries (LMICs) have a higher disease burden. ${ }^{1}$ In order for locally relevant health issues to be addressed in systematic reviews, local authors must be involved in conducting these reviews.

Cochrane is a world leader in the production of systematic reviews and an advocate for capacity building of individuals, groups and networks in LMICs. $^{2}$ One of Cochrane's capacity building goals is to ensure that people in LMICs have the necessary technological and methodological expertise to complete Cochrane reviews. However, other barriers may exist to conducting Cochrane reviews in LMICs. This may be because of the limited variety of review types published by Cochrane (intervention reviews, overviews of systematic reviews, diagnostic test accuracy reviews, methodology reviews, qualitative reviews and recently, prognostic reviews), difficulties forming review teams and delays in administrative processes. Previous research suggests that mentorship, practical assistance and dedicated time to complete reviews would benefit authors in LMICs. ${ }^{34}$

In 2017, Cochrane Africa (https://africa. cochrane.org/) was launched in response to the need to build local systematic review capacity and to promote the use of systematic review evidence in sub-Saharan Africa (SSA) ${ }^{5}$ Cochrane review production by Cochrane Africa is stifled by certain challenges that push competent Cochrane authors to publish 


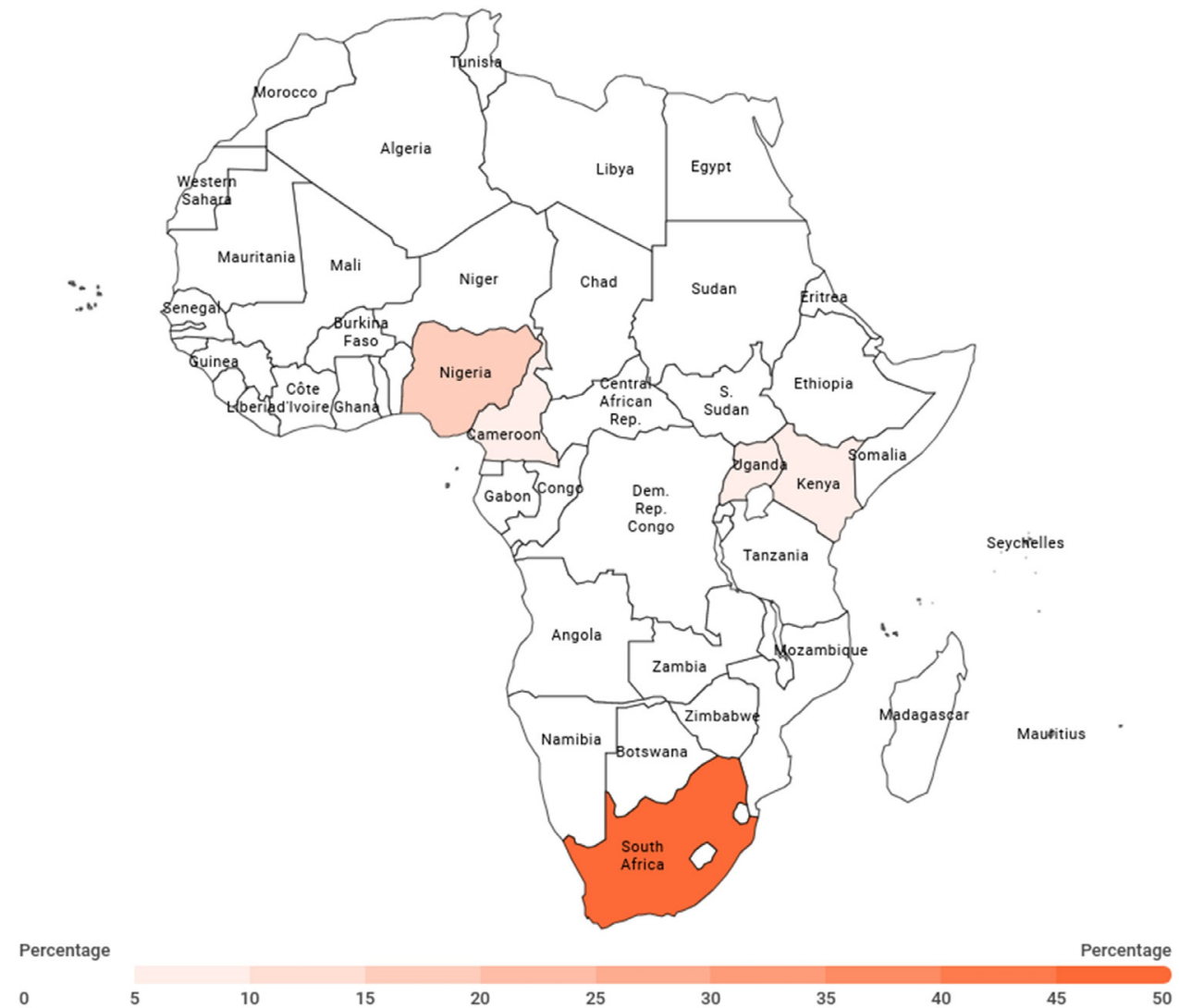

Figure 1 Distribution of Cochrane authors in sub-Saharan Africa (excluding Western Sahara, Morocco, Algeria, Tunisia, Libya and Egypt).

non-Cochrane reviews. To maximise existing capacity and to attract and retain more authors of Cochrane reviews, it is important to understand the publication practices of Cochrane SSA authors.

Our objectives were to investigate the extent to which Cochrane authors from SSA publish Cochrane and nonCochrane reviews, whether this has changed over time, and what topics and types of reviews have been published.

\section{METHODS}

\section{Patient and public involvement}

No patient involved

\section{Design}

We investigated publishing practices among SSA Cochrane authors. Using Archie, Cochrane's information management system (https://archie.cochrane.org/), we identified all Cochrane authors who reside or have an affiliation in a SSA country (in Archie) as of 10 April 2019. Archie includes a record of all individuals who have registered a systematic review topic. This database includes information on the stage of the systematic review - from protocol registration to review completion, the affiliation of the author, country and Cochrane Review Group to which the review belongs. A Cochrane author was defined as anyone who had a record in Archie linked to authorship of a Cochrane review. We then searched PubMed to identify systematic reviews and overviews on human health published by these authors (ie, we performed a search linking each SSA Cochrane author with our systematic review search string below) between 01 January 2008 and 18 April 2019. Eligible systematic reviews and overviews had predetermined objectives, eligibility criteria, at least two databases searched, data extraction and quality assessment. ${ }^{6}$ We excluded protocols, withdrawn systematic reviews and systematic reviews that were not on human health (eg, animal reviews, methods papers). Our PubMed search strategy was as follows:

"\#1 (( Systematic[sb] OR systematic review*[tiab] OR meta-analys*[tiab] OR metaanalys*[tiab]) NOT (animals[mh] NOT humans[mh]))) AND ("2008/01/01"[Date - Publication]: "2019/04/18"[Date - Publication]); \#2 surname initial [AU]; \#3 \#1 AND \#2"

All references were processed using Endnote reference management software (https://endnote.com/). After removing the duplicates, the full texts were retrieved and uploaded to DistillerSR (https://www.evidencepartners. $\mathrm{com} /$ products/distillersr-systematic-review-software/) for screening and data extraction.

Using the author list derived from Archie, we selected the articles in which the first or last author were from SSA. We selected these articles because we assumed that in first or last authorship roles, the authors played a significant role in determining whether it would be a Cochrane review or not.

We extracted details on country of origin, year of publication, whether it was a Cochrane or non-Cochrane 


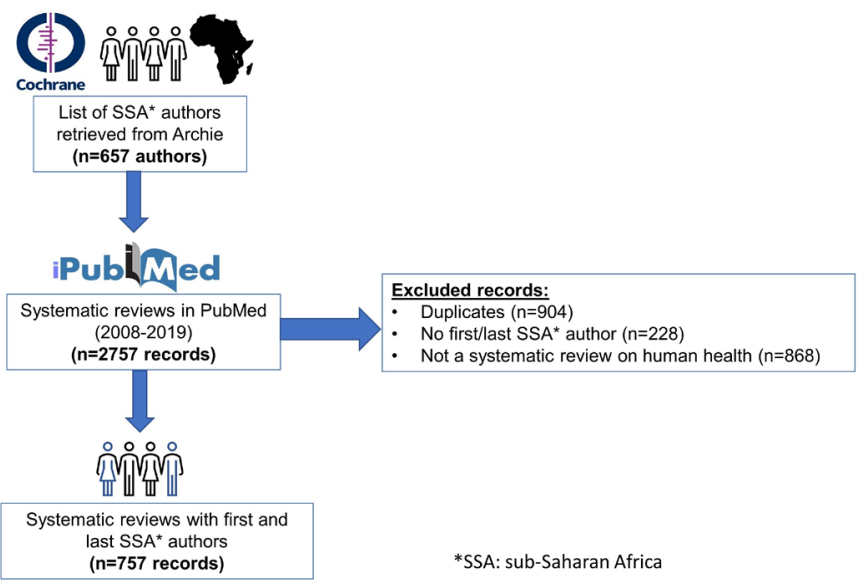

Figure 2 Flow of article selection.

review, the type of review (eg, intervention, diagnostic, qualitative) and the health topic (eg, infectious disease, public health) of the review. Reviewers categorised the studies based on the list of topics in the Cochrane Library (https://www.cochranelibrary.com/browse-by-topic). Two reviewers extracted data and a third reviewer adjudicated disagreements.

We described data using counts and percentages. All analyses were conducted using SPSS V.26.0.

\section{RESULTS}

\section{SSA Cochrane authors}

We identified 657 Cochrane authors from 19 of 48 SSA countries $(39.5 \%)$. Most authors were from South Africa $(\mathrm{n}=332 ; 51 \%)$, followed by Nigeria $(\mathrm{n}=126 ; 19 \%)$, Kenya $(\mathrm{n}=38 ; 6 \%)$, Uganda $(\mathrm{n}=33 ; 5 \%)$ and Cameroon $(\mathrm{n}=29$; $4 \%)$. Twenty-one (3\%) SSA Cochrane authors either came from (or reside in) a HIC but had an affiliation with a SSA country or were originally from a SSA country but work (or reside) in a HIC (see figure 1).

\section{Systematic reviews and overviews of systematic reviews}

Applying this list of SSA Cochrane authors in PubMed, we retrieved 2757 articles. After removing 904 duplicates, we screened the titles and abstracts of 1853 articles, of which 1096 were not eligible. A total of 757 articles were included, of which 53\% were non-Cochrane reviews and $47 \%$ were Cochrane reviews. The flow of article selection is shown in figure 2.

\section{Topics of research}

A total of 1136 topics were identified, with some reviews addressing more than one topic. The top five categories of topics addressed were infectious disease (24\%), pregnancy and childbirth (12\%), child health and neonatal care $(9 \%)$, health systems and services $(6 \%)$ and mental health $(6 \%)$. A full list of all the topics covered is shown in table 1.
Table 1 List of health topics covered in the included reviews

\begin{tabular}{|c|c|}
\hline Health topic & No (per cent) ${ }^{\star}$ \\
\hline Infectious disease & $275(24.2)$ \\
\hline Pregnancy and childbirth & $131(11.5)$ \\
\hline Child health and neonatal care & $104(9.2)$ \\
\hline Health systems and services & $71(6.3)$ \\
\hline Mental health & $66(5.8)$ \\
\hline Public health & $43(3.8)$ \\
\hline Heart and circulation & $42(3.7)$ \\
\hline Neurology & $31(2.7)$ \\
\hline Gynaecology & $29(2.6)$ \\
\hline Blood disorders & $26(2.3)$ \\
\hline Malnutrition and obesity & $26(2.3)$ \\
\hline Tobacco, drugs and alcohol & $24(2.1)$ \\
\hline Dentistry and oral health & $23(2)$ \\
\hline Surgery & $21(1.8)$ \\
\hline Lungs and airways & $20(1.8)$ \\
\hline Other & $19(1.7)$ \\
\hline Cancer & $18(1.6)$ \\
\hline Trauma and emergency medicine & $18(1.6)$ \\
\hline Pain and anaesthesia & $17(1.5)$ \\
\hline Endocrine and metabolic & $16(1.4)$ \\
\hline Orthopaedics & $15(1.3)$ \\
\hline $\begin{array}{l}\text { Complementary and complementary } \\
\text { medicine }\end{array}$ & $13(1.1)$ \\
\hline Skin disorders & $13(1.1)$ \\
\hline Health professional education & $12(1.1)$ \\
\hline Eyes and vision & $11(1)$ \\
\hline Ear, nose and throat & $8(0.7)$ \\
\hline Genetic disorders & $8(0.7)$ \\
\hline Kidney disease & $8(0.7)$ \\
\hline Gastroenterology and hepatology & $7(0.6)$ \\
\hline Urology & $5(0.4)$ \\
\hline $\begin{array}{l}\text { Consumer and communication } \\
\text { strategies }\end{array}$ & $4(0.4)$ \\
\hline Health and safety at work & $4(0.4)$ \\
\hline Wound & $4(0.4)$ \\
\hline Nursing care & $2(0.2)$ \\
\hline Allergy and intolerance & $1(0.1)$ \\
\hline Rheumatology & $1(0.1)$ \\
\hline Total & $1136(100.0)$ \\
\hline
\end{tabular}

${ }^{*}$ Count of topics covered in 757 systematic reviews.

\section{Type of research}

Close to three-quarters $(71 \%)$ of the included articles were systematic reviews of interventions, followed by systematic reviews of risk factors $(10 \%)$, systematic reviews of prevalence $(7 \%)$, diagnostic test accuracy reviews 


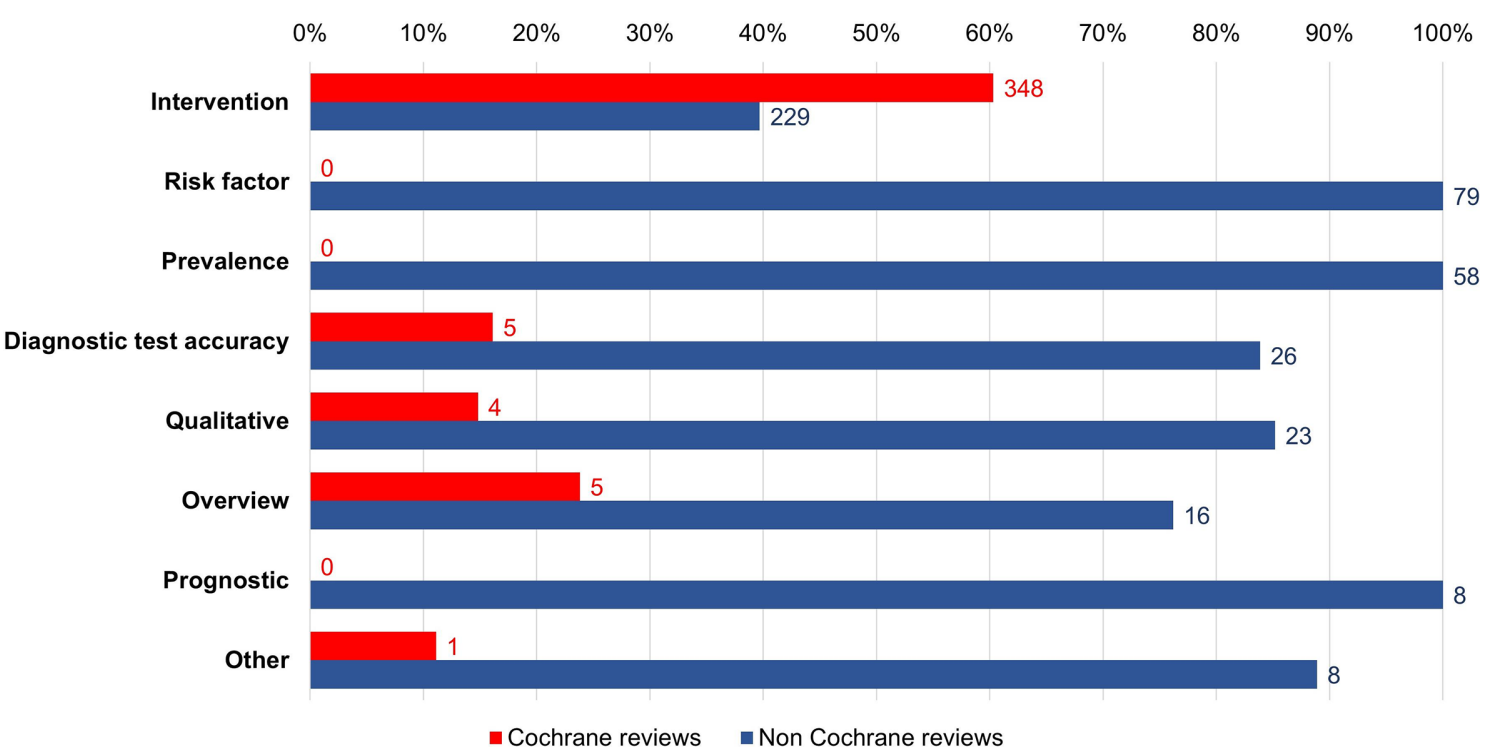

Figure 3 Types of Cochrane and non-Cochrane reviews published by Cochrane SSA authors. SSA, sub-Saharan Africa.

$(4 \%)$, qualitative reviews $(3 \%)$, overviews $(3 \%)$, prognostic reviews $(1 \%)$ and others (eg, reviews addressing cost-effectiveness, disease patterns; $1 \%$ ).

The intervention reviews were more likely to be Cochrane reviews $(60.3 \%$ vs $39.7 \%)$. Conversely, the overviews $(23.8 \%$ vs $76.2 \%)$, the qualitative reviews (14.8\% vs $85.2 \%)$, the diagnostic test accuracy reviews $(16.1 \%$ vs $83.9 \%)$ and the 'other' reviews $(11.1 \%$ vs $88.9 \%$ ) were more likely to be non-Cochrane reviews. All the prognostic, prevalence and risk factor reviews were non-Cochrane reviews. Figure 3 illustrates the types of Cochrane and non-Cochrane reviews published by SSA Cochrane authors.

\section{Trends in Cochrane and non-Cochrane reviews}

Between 2008 and 2018, the number of non-Cochrane reviews increased steadily from 14 per year to 61 per year, while the number of Cochrane reviews only increased from 16 per year to 31 per year in the same period (see figure 4).

\section{DISCUSSION}

In this bibliometric analysis of publication practices of systematic reviews among SSA Cochrane authors, we

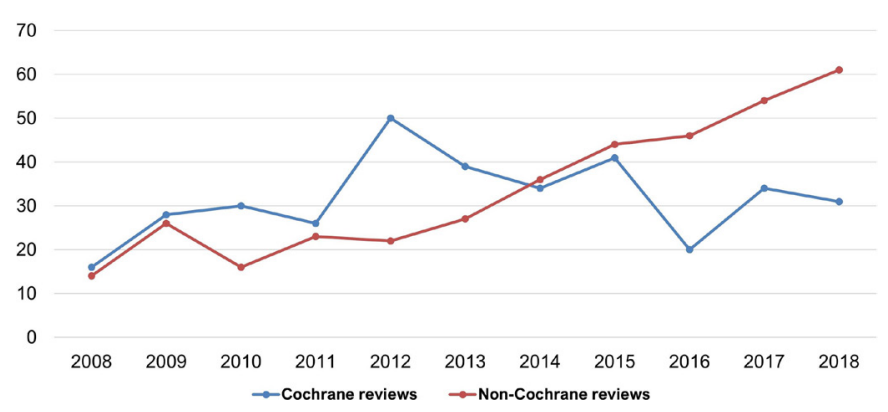

Figure 4 Trends in publication of Cochrane and nonCochrane review among SSA Cochrane authors. SSA, subSaharan Africa. found that South Africa and Nigeria contributed the most reviews. Of these, most were intervention reviews, which were more likely to be Cochrane reviews. The nonintervention reviews were more likely to be published in non-Cochrane journals. Furthermore, over the study period, there has been a relative decrease in the number of Cochrane reviews published by Cochrane SSA authors, with a corresponding increase in the number of nonCochrane reviews. About a quarter of the reviews covered infectious disease topics.

These findings have important implications. First, it shows the countries in SSA for which more Cochrane outreach is needed to enhance evidence synthesis production and, ultimately, evidence informed decision making. It also shows which countries have the potential to offer leadership and capacity building. At the time of the study, South Africa and Nigeria have the only registered geographical centres in $\mathrm{SSA}^{5}$ and have jointly contributed to substantial amounts of capacity building for reviews in SSA. It is important to build the capacity for systematic reviews in other SSA countries where capacity for conducting reviews lags. ${ }^{1}$

Second, the limited diversity in types of Cochrane reviews might explain why authors trained in Cochrane methods would prefer non-Cochrane reviews. During the study period, the Cochrane Library mainly had reviews of interventions, diagnostic accuracy, methodology, qualitative and overviews published, as well as a few prognostic reviews. As such, other types of reviews such as prevalence or risk factor reviews would have to be submitted elsewhere. Likewise, for diagnostic test accuracy reviews, the analytical capabilities of Cochrane's software, Review Manager (RevMan, https://training. cochrane.org/online-learning/core-software-cochranereviews/revman) are limited. ${ }^{7}$ For example, RevMan does not have the capacity for bivariate modelling required for diagnostic accuracy meta-analysis, and does not perform meta-analysis of proportions. In the past year, additional 
review types have been added, (eg, scoping reviews and rapid reviews) largely in response to the need for reviews at pace during the COVID-19 pandemic. This may increase the interest of authors to publish within Cochrane to answer locally relevant questions using Cochrane methods and infrastructure.

As review authors gain experience with systematic reviews and overviews, the often lengthy and burdensome editorial process associated with some Cochrane review groups may cause authors to choose easier and quicker routes. Previous literature suggests that nonCochrane systematic reviews may be preferred if the authors lacked knowledge of Cochrane processes and, the time required to complete a Cochrane review, or sought to publish in a journal more directly relevant to the topic of interest. ${ }^{8}$ While the Cochrane editorial processes may lead to higher quality products, ${ }^{9}{ }^{10}$ more conservative results, ${ }^{11}$ and more transparent declaration of conflicts of interest, ${ }^{12}$ reviews authors may be working with shorter timelines (eg, as expected from funders or decision-makers) and thus seek alternative journals. Furthermore, the downward trend in publication of Cochrane reviews may be a reflection of other longstanding issues with research output in collaborative health research, including limited opportunities with regards to research funding; academic resources; stronger, deeper and wider research networks; and editorial roles for authors from low-income countries. ${ }^{13-15}$ Although these issues have not been extensively studied with systematic reviews, if such power imbalances exist within Cochrane, it may explain why the number of first or last SSA authors reduced during the study period.

For Cochrane-trained authors, publishing systematic reviews outside of Cochrane may not be a bad thing. It may allow for more methodological flexibility, the opportunity to access diverse funding sources (including industry funds) and expand their academic networks beyond Cochrane. This expansion of choice of journals may also be indicative of the role of Cochrane-trained authors influencing evidence syntheses beyond Cochrane.

Infectious disease research appears to be a prime focus of SSA research. This is not surprising given the disproportionate burden of infectious disease borne by SSA, and this focus may be further spurred by the availability of funds for infectious disease research. ${ }^{16}$

This work is not without limitations. First, we assumed that the first and last authors would be most influential in determining whether the review will be a Cochrane review or not. This may not always be the case. Second, bibliometric analyses alone cannot fully explain author behaviours or preferences, and further research is warranted to understand motivations. Third, the definition of 'review' used in this study is one of many, ${ }^{17}$ and less stringent definitions might yield a larger number of non-Cochrane reviews.

This work has informed the development of a mixedmethods study on the publication practices of Cochrane authors in SSA, for which data collection is ongoing.

\section{CONCLUSION}

Our findings suggest that after receiving training or resources in Cochrane review methodology, authors in SSA are increasingly diversifying the kinds of reviews they publish, using non-Cochrane journals. Systematic review and overview production in SSA is geographically skewed, with most reviews coming from one country and almost all reviews coming from a handful of countries. A deeper understanding of what motivates authors to select journals would inform capacity building initiatives, the choice of the most appropriate journal and the growth of Cochrane in SSA.

\section{Author affiliations}

${ }^{1}$ Health Research Methods, Evidence and Impact, McMaster University Faculty of Science, Hamilton, Ontario, Canada

${ }^{2}$ Biostatistics Unit, The Research Institute of St Joe's Hamilton, Hamilton, Ontario,

Canada

${ }^{3}$ Centre for the Development of Best Practices in Health, Yaounde, Cameroon ${ }^{4}$ Department of Global Health, Stellenbosch University, Cape Town, South Africa ${ }^{5}$ Centre for Evidence-based Health Care, Department of Global Health, Stellenbosch University Faculty of Medicine and Health Sciences, Cape Town, South Africa

${ }^{6}$ Cochrane South Africa, South African Medical Research Council, Cape Town, South Africa

${ }^{7}$ Cochrane Nigeria, Institute of Tropical Diseases Research and Prevention, University of Calabar Teaching Hospital, Calabar, Nigeria

Twitter Tamara Kredo @tamarakredo, Charles Shey Wiysonge @CharlesShey and Taryn Young @TarynYoung3

Acknowledgements Anel Schoonees, Taryn Young, Solange Durao and Tamara Kredo are partly supported by the Research, Evidence and Development Initiative (READ-It) project. READ-It (project number 300342-104) is funded by UK aid from the UK Government; however, the views expressed do not necessarily reflect the UK Government's official policies.

Contributors AS and LM conceived of the study. AS, JO and LM developed the methods with input from all authors. J0 ran the searches. LM, AS, JO, DA, SD, EE, AH, TK, CSW and TY extracted data and performed consensus exercises. AS and LM analysed the data. AS and LM wrote the first draft. LM, AS, JO, DA, SD, EE, AH, TK, CSW and TY revised several versions of the manuscript. All authors reviewed and approved the final draft.

Funding The authors have not declared a specific grant for this research from any funding agency in the public, commercial or not-for-profit sectors.

Map disclaimer The inclusion of any map (including the depiction of any boundaries therein), or of any geographic or locational reference, does not imply the expression of any opinion whatsoever on the part of BMJ concerning the legal status of any country, territory, jurisdiction or area or of its authorities. Any such expression remains solely that of the relevant source and is not endorsed by BMJ. Maps are provided without any warranty of any kind, either express or implied.

\section{Competing interests None declared.}

Patient and public involvement Patients and/or the public were not involved in the design, or conduct, or reporting, or dissemination plans of this research.

Patient consent for publication Not applicable.

Ethics approval This is a bibliometric analysis of publicly available data. Ethics approval was not required.

Provenance and peer review Not commissioned; externally peer reviewed.

Data availability statement No data are available. № additional data available.

Open access This is an open access article distributed in accordance with the Creative Commons Attribution Non Commercial (CC BY-NC 4.0) license, which permits others to distribute, remix, adapt, build upon this work non-commercially, and license their derivative works on different terms, provided the original work is properly cited, appropriate credit is given, any changes made indicated, and the use is non-commercial. See: http://creativecommons.org/licenses/by-nc/4.0/. 


\section{ORCID iDs}

Lawrence Mbuagbaw http://orcid.org/0000-0001-5855-5461

Tamara Kredo http://orcid.org/0000-0001-7115-9535

Charles Shey Wiysonge http://orcid.org/0000-0002-1273-4779

\section{REFERENCES}

1 Oliver S, Bangpan M, Stansfield C, et al. Capacity for conducting systematic reviews in low- and middle-income countries: a rapid appraisal. Health Res Policy Syst 2015;13:23.

2 Young T, Garner P, Kredo T. Cochrane and capacity building in lowand middle-income countries: where are we at? Cochrane Database Syst Rev 2013:11:ED000072.

3 Oliver J, Young T. What can the Cochrane collaboration do to support people living in developing countries? A survey. Corroboree Abstracts of the 13th Cochrane Colloquium 2005:22-6.

4 Oliver J, Kredo T, Zani B. Barriers and facilitators to completing a cochrane review: a survey of authors in the African region. 21st Cochrane Colloquium; 2013 19-23 September; Quebec City, Canada.

5 Mbuagbaw L, Zogo PO, Kredo T, et al. Cochrane Africa: a network of evidence-informed health-care decision making across sub-Saharan Africa. Pan Afr Med J 2018;29:196.

6 Young T, Rohwer A, Volmink J, et al. What are the effects of teaching evidence-based health care (EBHC)? overview of systematic reviews. PLoS One 2014;9:e86706.

7 Collaboration C. Review manager (RevMan)[computer program]: Version 2014

8 Hartling L, Shave K, Thomson D, et al. Publication of reviews synthesizing child health evidence (PORSCHE): a survey of authors to identify factors associated with publication in Cochrane and nonCochrane sources. Syst Rev 2016;5:104.

9 Olsen O, Middleton P, Ezzo J, et al. Quality of Cochrane reviews: assessment of sample from 1998. BMJ 2001;323:829-32.

10 Page MJ, Shamseer L, Altman DG, et al. Epidemiology and reporting characteristics of systematic reviews of biomedical research: a cross-sectional study. PLoS Med 2016;13:e1002028.

11 Useem J, Brennan A, LaValley M, et al. Systematic differences between Cochrane and Non-Cochrane meta-analyses on the same topic: a matched pair analysis. PLoS One 2015;10:e0144980.

$12 \mathrm{Yu}$ J, Su G, Hirst A, et al. Identifying competing interest disclosures in systematic reviews of surgical interventions and devices: a crosssectional survey. BMC Med Res Methodol 2020;20:260.

13 Hedt-Gauthier BL, Jeufack HM, Neufeld NH, et al. Stuck in the middle: a systematic review of authorship in collaborative health research in Africa, 2014-2016. BMJ Glob Health 2019;4:e001853.

14 Kelaher M, Ng L, Knight K, et al. Equity in global health research in the new millennium: trends in first-authorship for randomized controlled trials among low- and middle-income country researchers 1990-2013. Int J Epidemiol 2016;45:2174-83.

15 Smith E, Hunt M, Master Z. Authorship ethics in global health research partnerships between researchers from low or middle income countries and high income countries. BMC Med Ethics 2014;15:42.

16 Bhutta ZA, Sommerfeld J, Lassi ZS, et al. Global burden, distribution, and interventions for infectious diseases of poverty. Infect Dis Poverty 2014;3:21.

17 Krnic Martinic M, Pieper D, Glatt A, et al. Definition of a systematic review used in overviews of systematic reviews, metaepidemiological studies and textbooks. BMC Med Res Methodol 2019;19:203. 